2. Birth weight varies from $1.01 \mathrm{~kg}$ to $2.80 \mathrm{~kg}$

3. Out of 20 preterm babies $17(85 \%)$ had some form of pain relief.

4. 3(15\%) of babies had received no pain control measures.

5. Non pharmacological measures were used in $15(75 \%)$ of babies.

6. Pharmacological measures were used in 13(65\%) babies.

7. Oral sucrose was used in $10(50 \%)$ babies.

8. 3(15\%) babies received topical analgesia for ROP screening.

9. Combined methods of pharmacological and non pharmacological pain relief were used in $13(65 \%)$ babies.

Conclusions Overall pain management was was not satisfactory in our unit.

Oral sucrose was used only in 50 per cent of the babies and also it was used inappropriately.In most of the babies it was given just before procedure or during the procedure. There were no records found for how many doses of oral sucrose given to any neonate in twenty four hours.

While giving vitamin $\mathrm{K}$ injection to babies they received no pain relief.

The use of combination methods of pain relief was relatively low.

Recommendations and action plan

Oral sucrose should be given at least 2 minutes before the procedure as per recommendations.

We can do better by following the use of combination methods.

While doing any procedure there should be documentation of pain relieving methods followed along with procedure notes.

It is also better to keep record of number of doses of sucrose given to any pre term baby in 24 hours.

\section{P461 THE DIGITS DON'T ADD UP: EXOMPHALOS AND DIGITAL DYSPLASIA}

Zaheera Yusuf*, Michael Boyle. Rotunda Hospital, Dublin, Ireland

10.1136/archdischild-2019-epa.797

Baby Girl S was born at term by elective caesarean section for an antenatal diagnosis of exomphalos. At delivery she was noted to have exomphalos major with distal dysplasia of the second, third, and fourth digits on her left hand. X-ray confirmed the second and third digit to have only two ossified phalanges, and the fourth digit to have only one ossified phalanx. On review of digital anomalies and exomphalos we found that digital dysplasia had not been previously described in infants with exomphalos.

Exomphalos is a midline abdominal wall defect occurring at the base of the umbilical cord affecting about 1 in 5000 pregnancies. ${ }^{1}$ The defect contains abdominal viscera and is covered by a membrane of amnion, peritoneum, and Wharton's jelly. ${ }^{2}$ Exomphalos can be further classified as exomphalos minor, where the opening is less than $4 \mathrm{~cm}$ and only contains the intestine, and as exomphalos major, where the opening is greater than $4 \mathrm{~cm}$ and/or with the liver inside the cord. ${ }^{3}$ Unlike gastroschisis, exomphalos has a membrane covering the abdominal contents, and is more likely to be part of a syndrome, or have associated anomalies. ${ }^{2}$ Approximately half of infants have associated anomalies, including Trisomy 13 and
18, syndromes such as Beckwith-Wiedemann and Pentalogy of Cantrell, cardiac anomalies, pulmonary hypoplasia, VACTERL anomalies, and nervous system abnormalities such as holoprosencephaly and anencephaly. ${ }^{1,2,3}$

Baby Girl $S$ did not require resuscitation at delivery and the sac was intact. She was transferred to a tertiary paediatric hospital for surgical management. Her neonatal course was uneventful except for three moderate hypoglycaemic episodes. She had imaging, a cardiology review, and a genetics review. Her microarray and karyotype were normal, and she was believed to be non-syndromic with no other associated anomalies. She did not meet criteria for Beckwith-Wiedemann syndrome, despite the episodes of hypoglycaemia. Surgical management of the exomphalos involved a silastic pouch to allow gradual reduction of the defect.

This is the first documented case of digital dysplasia with exomphalos.

\section{REFERENCES}

1. Southampton Children's Hospital. Exomphalos. 2014. Accessed at http://www. uhs.nhs.uk/OurServices/Childhealth/Neonatalsurgery/Conditionswetreat/Exomphalos. aspx

2. Neonatal Directorate Management Committee. Clinical Practice Guideline: Exomphalos (Omphalocele). Government of Western Australia North Metropolitan Health Service. 1 September 2014. https://www.kemh.health.wa.gov.au/ /media/ Files/Hospitals/WNHS/For\%20health\%20professionals/Clinical\%20guidelines/NEO/ WNHS.NEO.ExomphalosOmphalocele.pdf

3. Great Ormond Street Hospital for Children. Exomphalos. 2018. Accessed at https://www.gosh.nhs.uk/conditions-and-treatments/conditions-we-treat/ exomphalos

\section{P462 OUT OF HOUR BLOOD TRANSFUSIONS IN NICU AND CAN IT BE PREVENTED?}

Navdeep Kaur Brar*, Prof Naomi Mccallion. The Rotunda Hospital , Dublin, Ireland

10.1136/archdischild-2019-epa.798

Introduction Overnight blood transfusion carry increased risk of transfusion complication due to availability of less staff to monitor the patient and there is likely to be fewer medical and laboratory staff available to respond to the complication. As per hospital protocol non urgent red blood transfusions should be done during working hours so that there is less work load on night duty doctor, nurses and lab personnel. Other reason to order transfusion during working hours so that out of hours charges should be avoidable which is 150 Euros, as not always blood packs supply is available in the hospital.The aim of the audit to know why red blood cell transfusion top- ups were ordered out of hours and to see how can it can be avoided, thereby saving cost.

Methods Data was collected retrospectively from July 2017 till September 2017. The total of 77 transfusions took place on 26 patients as some patient had multiple transfusions. The collected data was entered directly into audit tool on excel sheet. The questionnaire includes the following questions: gestation, date of birth, weight at delivery,reason for transfusion, general status, haemoglobin level at the time of transfusion, the time of FBC taken and resulted, in working hours or out of hours, the time when blood transfusion ordered and collected, in working hours or out of hours, day of life at transfusion. The charts of patients were collected and reviewed.The guidelines used for this audit was the Neonatal administration and transfusion specific blood/blood components 2016 issued by Rotunda Hospital 